\title{
Sulfide Catalysts Supported on Porous Aromatic Frameworks for Naphthalene Hydroprocessing
}

\author{
Eduard Karakhanov ${ }^{1, *}$, Yulia Kardasheva ${ }^{1}$, Leonid Kulikov ${ }^{1}$, Anton Maximov ${ }^{1,2}$, \\ Anna Zolotukhina ${ }^{1}$, Maria Vinnikova ${ }^{1}$ and Andrey Ivanov ${ }^{2}$ \\ 1 Laborotory of Catalysis and Petrochemical Synthesis, Department of Petroleum Chemistry and \\ Organic Catalysis, Lomonosov Moscow State University, Moscow 119991, Russia; \\ yuskard@petrol.chem.msu.ru (Y.K.); mailforleonid@mail.ru (L.K.); max@ips.ac.ru (A.M.); \\ anisole@yandex.ru (A.Z.); mary.pk@mail.ru (M.V.) \\ 2 Institute of Petrochemical Synthesis, Russian Academy of Sciences, Moscow 119991, Russia; \\ aoivanov@ips.ac.ru \\ * Correspondence: kar@petrol.chem.msu.ru; Tel.: +7-495-939-53-77
}

Academic Editors: Jianguo Liu and Di-Jia Liu

Received: 30 June 2016; Accepted: 5 August 2016; Published: 16 August 2016

\begin{abstract}
This paper describes the first example of using porous aromatic frameworks as supports for sulfide catalysts for the hydrogenation of aromatic hydrocarbons. The synthesis of bimetallic Ni-W and Ni-Mo sulfides was performed by in situ decomposition of $\left[(\mathrm{n}-\mathrm{Bu})_{4} \mathrm{~N}_{2}\left[\mathrm{Ni}(\mathrm{MeS})_{2}\right](\mathrm{Me}=\mathrm{W}, \mathrm{Mo})\right.$ complexes, supported on mesoporous aromatic framework with a diamond-like structure. It is shown that the highest naphthalene conversions were achieved in the case of additional sulfidation with sulfur. After the reaction, catalysts were characterized by X-ray photoelectron spectroscopy and high-resolution transmission electron microscopy. The activity of synthesized catalysts has been studied using naphthalene as a model substrate. The materials used in this study were substantially active in hydrogenation and slightly in hydrocracking of naphthalene.
\end{abstract}

Keywords: heterogeneous catalysis; hydrogenation; nano-structured catalysts; porous aromatic frameworks

\section{Introduction}

One of the current problems of modern petrochemistry is the search for highly efficient catalysts for conversion of heavy hydrocarbons into light products by hydrocracking-hydrogenation reactions. A significant part of these hydrocarbons make up polycyclic aromatic hydrocarbons, such as naphthalene, anthracene, phenanthrene, and their derivatives. The traditional hydrogenationhydrocracking conditions include the usage of high pressures and temperatures and various sulfided catalysts that are resistant to poisoning by heteroatomic compounds. In particular, sulfides of Group VIB (molybdenum and tungsten) and Group VIII (cobalt, nickel) are of great industrial interest due to they are widely used in petroleum refining for hydroprocessing applications. Moreover, these sulfides are active in catalytic coal liquefaction [1], i.e., in breaking up macromolecules into low molecular weight fragments.

In general, two methods for obtaining sulfide catalysts are used: impregnation of different supports by metal salts with post-sulfidizing [2-5], and usage of metal sulfides as catalyst precursors with conversion to a catalytically-active form during the reaction [6-9]. The mechanisms, by which metal sulfides decompose to their active form were investigated in several studies [10,11]. It is well-known, that the NiMoS (or NiWS) phase could be considered as slabs or "sandwiches", where two sulfur compact planes are placed one over the other and form trigonal prismatic holes. Mo occupies half of these holes, whereas $\mathrm{Ni}$ is "decorating" the borders of the slab in the same plane formed by Mo [12,13]. 
The role of support interactions with NiMeS slabs is a key parameter used to control the catalyst activity and selectivity $[14,15]$. The support effect has been related to the formation of the Topsøe's Type I and Type II Ni-Mo-S structures. It was revealed that the Type II NiMoS structure is more active in hydroprocessing reactions than the Type I structure [16]. Importantly, the Type I phase predominates when NiMeS catalysts are supported on alumina or zeolites, i.e., oxide supports, whereas the Type II phase is the main phase in the case of carbon supports $[17,18]$. Thus, it is of interest to investigate the properties of NiMoS and NiWS catalysts supported by novel carbon-based materials.

Recently, new types of carbon-based materials, called porous aromatic frameworks (PAF), have been developed [19]. The advantages of PAFs are a regular, rigid, aromatic structure, high surface area, physicochemical stability, and facile functionalization. The most famous example of PAFs is PAF-1 with dia topology, which possesses an extremely high surface area of $5640 \mathrm{~m}^{2} \cdot \mathrm{g}^{-1}$ by Brunauer-Emmett-Teller (BET) theory. However, within the "homologous series" of PAFs with diamond-like topology the surface area decreases dramatically from $5640 \mathrm{~m}^{2} \cdot \mathrm{g}^{-1}$ for PAF-1 with two benzene rings at the edge to $740 \mathrm{~m}^{2} \cdot \mathrm{g}^{-1}$ for PAF-11 with four benzene rings at the edge [20]. At the same time, PAF-1 is a microporous material, whereas PAF-11 presents an example of mesoporous material. The representative structure of porous aromatic framework with dia topology is presented in the Figure 1.

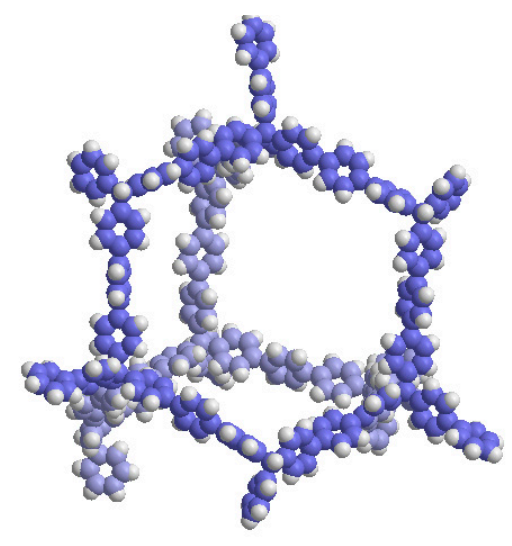

Figure 1. The representative structure of porous aromatic framework with dia topology; three benzene rings in the edge.

Formerly, we reported the size-selective hydrogenation of aromatic compounds on the ruthenium nanoparticles supported by PAFs [21]. The hydrogenation of very large molecules, such as tetralin, was ineffective on a catalyst based on the framework with lesser pore size. Therefore, in order to implement hydrocracking-hydrogenation reactions of heavy hydrocarbons the mesoporous frameworks should be used.

Herein, we describe the usage of PAFs as support for NiMoS and NiWS catalysts. The original idea is to realize a slurry process. We believe that porous aromatic frameworks will be appropriate materials for obtaining NiMeS nanoparticles with a Type II structure due to their advantages mentioned above. The precursors for synthesis of bimetallic Ni-W and Ni-Mo sulfides were prepared through impregnation of $\left[(\mathrm{n}-\mathrm{Bu})_{4} \mathrm{~N}_{2}\left[\mathrm{Ni}\left(\mathrm{MeS}_{4}\right)_{2}\right](\mathrm{Me}=\mathrm{W}, \mathrm{Mo})\right.$ with PAFs in THF and subsequent evaporation of the solvent. The formation of active phase occurred in situ during the reaction of hydrotreatment of naphthalene, used as the model molecule of condensed aromatics.

\section{Results and Discussion}

\subsection{Synthesis of Precursors and Characterization of Catalysts}

Through a simple impregnation of PAF-20 or PAF-30 with $\left[(\mathrm{n}-\mathrm{Bu})_{4} \mathrm{~N}\right]_{2}\left[\mathrm{Ni}\left(\mathrm{MeS}_{4}\right)_{2}\right](\mathrm{Me}=\mathrm{W}, \mathrm{Mo})$ and further drying under reduced pressure, PAF-NiWS and PAF-NiMoS precursors were obtained. 
We assume that contact between nickel, sulfur, and molybdenum or tungsten in the initial complex would increase the content of mixed Ni-Mo-S and Ni-W-S phases in the resulting catalysts.

The successful infiltration of metal salts into the PAFs could be shown by photographic pictures (Figure 2). The color of PAF changed from off-white to dark-green in case of NiMoS catalyst and to dark-orange in the case of NiWS catalyst. No explicit heterogeneity was revealed in the obtained powders.

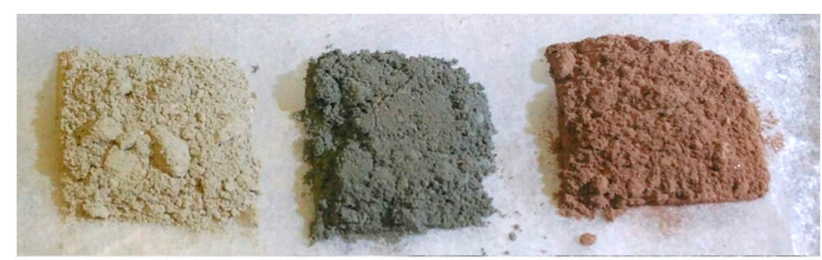

\section{PAF PAF-NiMoS PAF-NiWS}

Figure 2. Photographic picture of porous aromatic framework infiltrated with different bimetallic sulfides.

The mechanisms of thermal decomposition of alkylammonium thiomolybdates and thiotungstates were considered earlier [11]. Generally, the thermal degradation occurs in a wide temperature range (130-360 $\left.{ }^{\circ} \mathrm{C}\right)$, leading to several metal sulfides. However, decomposition of carbon-rich thiosalts, like tetrahexylammonium and tetraheptylammonium thiotungstates, leads to sulfur-deficient products and increasing of carbon content in residue. It is assumed that atoms of sulfur could be replaced by carbon atoms in the $\mathrm{WS}_{2}$ phase formation process [22]. Furthermore, in the presence of water the main products of thermal decomposition are mixed oxysulfides, MeOxSy. These phases possess less activity in hydrotreatment reactions, therefore, it is desirable to prevent their formation or to convert them to a more active condition. Probably, one of the easiest ways to overcome these difficulties is to use sulfidizing agent. In this regard, the catalytic performance of catalysts both in the presence of sulfur and without it was investigated.

Figure $3 \mathrm{a}, \mathrm{b}$ show representative high-resolution transmission electron microscopy micrographs of NiMeS catalysts. Molybdenum sulfide and tungsten sulfide lamellar structures are observed in all cases. The black, thread-like fringes are the $\mathrm{MoS}_{2}$ or $\mathrm{WS}_{2}$ slabs. For each crystallite the number $\mathrm{N}$ of three-dimensional stacked layers and their length $L$ have been considered. The distributions of slab lengths and stacking number are presented in Figure $3 c-f$. The distances between the fringes were calculated to be $0.63 \mathrm{~nm}$ in the case of molybdenum catalysts, and $0.65 \mathrm{~nm}$ in the case of tungsten catalysts, which is consistent with the interplanar spacing for bulk $\mathrm{MoS}_{2}$ and $\mathrm{WS}_{2}$ reported in the literature $[4,12,23]$.

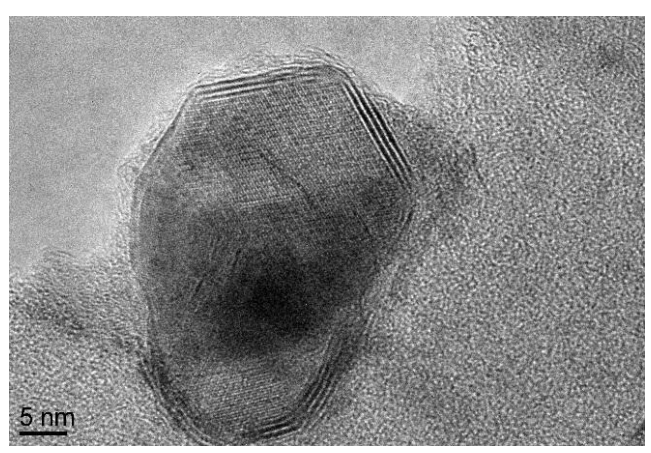

(a)

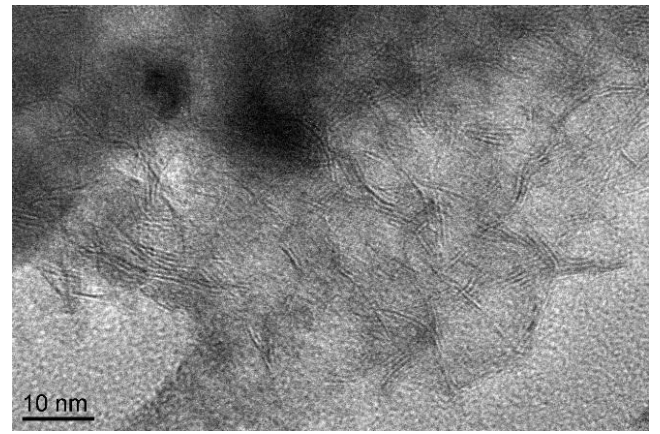

(b)

Figure 3. Cont. 


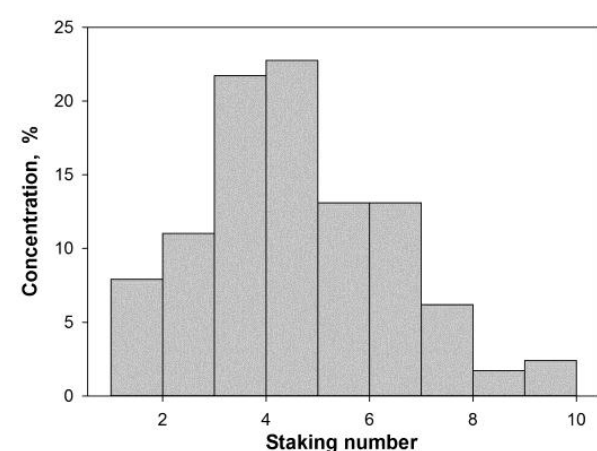

(c)

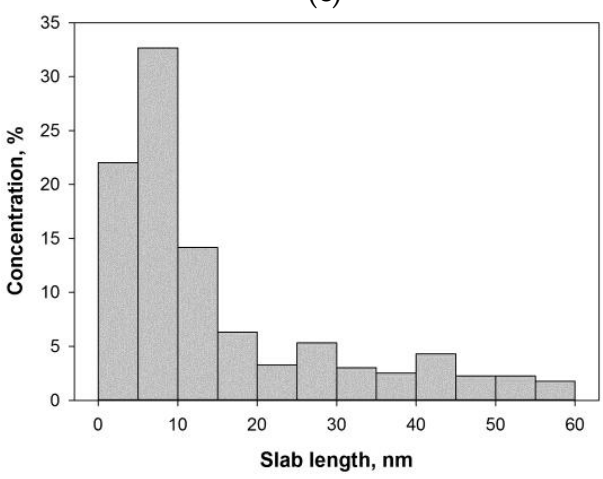

(e)

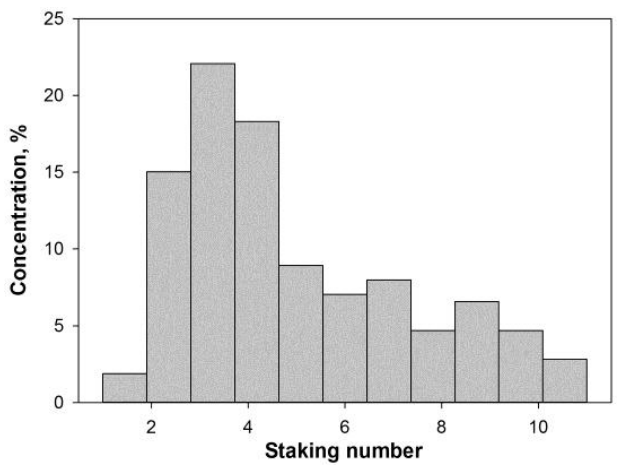

(d)

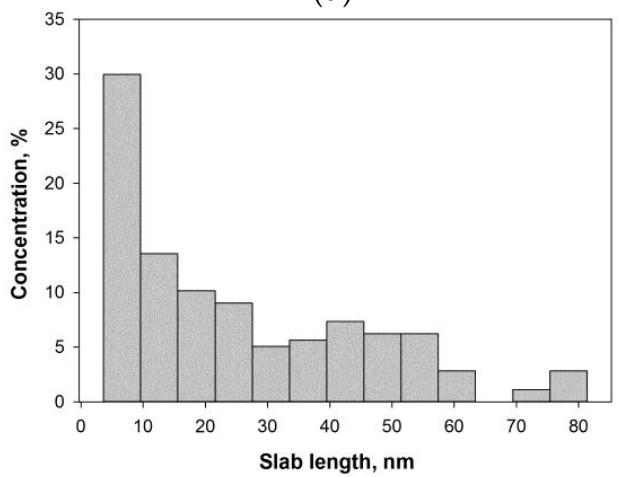

(f)

Figure 3. Representative micrographs of the catalysts: (a) PAF-NiWS; (b) PAF-NiMoS; distribution of particles with according to their length and stacking number of slabs: (c,e) for PAF-NiWS; $(\mathbf{d}, \mathbf{f})$ for PAF-NiMoS.

The average dimensions of the NiMeS phase particles and the average stacking number in the $\mathrm{MeS}_{2}$ crystallites are presented in Table 1.

Table 1. Textural characteristics of the metal sulfides.

\begin{tabular}{ccccc}
\hline Catalyst & $\overline{\boldsymbol{L}}, \mathbf{n m}$ & $\overline{\boldsymbol{N}}$ & $\boldsymbol{D}$ & $f_{\boldsymbol{e}} / f_{\boldsymbol{c}}$ \\
\hline PAF-NiMoS & $25.5 \pm 16.9$ & 4.9 & 0.031 & 38.3 \\
PAF-NiWS & $15.2 \pm 11.3$ & 4.4 & 0.037 & 22.3 \\
\hline
\end{tabular}

The calculation of $\mathrm{MoS}_{2}$ and $\mathrm{WS}_{2}$ dispersion, was performed using Equation (1), which measure the average fraction of metal atoms at the $\mathrm{MeS}_{2}$ edge surface under the assumption that sulfide slabs present perfect hexagons:

$$
D=\frac{M_{e d g}+M_{c r n}}{M_{t o t}}=\frac{\sum_{i=1 \ldots . t} 6 n_{i}-6}{\sum_{i=1 \ldots t} 3 n_{i}^{2}-3 n_{i}+1}
$$

where $M_{\text {edg }}$ is the number of metal atoms at the edge surface, $M_{\text {cor }}$ is the number of metal atoms at the corner sites, $M_{\text {tot }}$ is the total number of metal atoms, $n_{i}$ is the number of metal atoms along one side of the sulfide slab, determined by its length, and $t$ is the total number of slabs in the microphotograph.

The number of slabs per stack was determined to obtain the average staking degree:

$$
\bar{N}=\frac{\sum_{i=1 \ldots t} n_{i} N_{i}}{\sum_{i=1 \ldots .} n_{i}}
$$


where $n_{i}$ is the number of stacks in $N_{i}$ layers. The edge-to-corner ratio of a sulfide slab $f_{e} / f_{c}$ was calculated using the following equation $[24,25]$ :

$$
\frac{f_{e}}{f_{c}}=\frac{M_{e d g}}{M_{c r n}}=\frac{10 \bar{L} / 3.2-2}{2}
$$

where $\bar{L}$ is the average slab length (nm).

The internal microstructure of catalysts was examined by selected area electron diffraction (SAED) analysis and X-ray photoelectron spectroscopy analysis. The typical SAED pattern of catalysts is presented in Figure 4. Intensive (100), (103), and (110) orientation planes of the $\mathrm{WS}_{2}$ phase indicate multiple dimensional ordering—both in-plane and vertical—of nanoparticles.
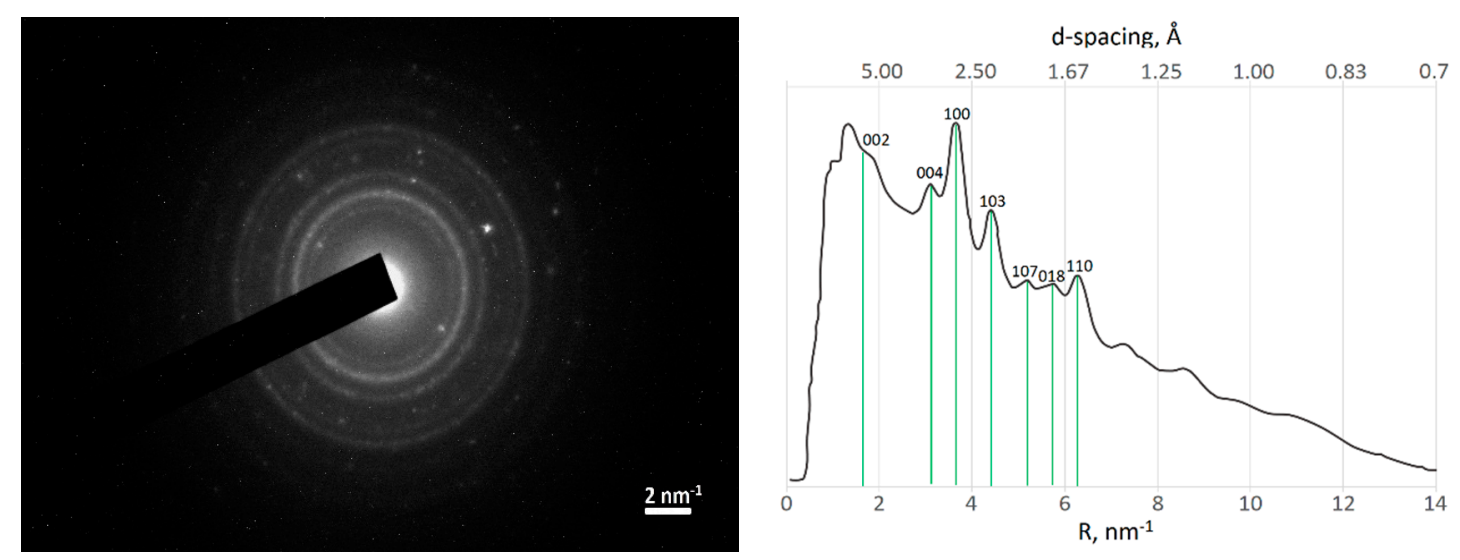

Figure 4. Selected area electron diffraction (SAED) pattern of the PAF-20-NiWS catalyst after reaction in the presence of sulfur.

XPS analysis of the samples revealed that all the catalysts exhibit peaks characteristic of carbon, sulfur, tungsten, and oxygen (Figure 5a-c). The absence of nickel signals in spectra could be attributed to full decoration of nickel atoms. All the spectra contain three forms of metal: sulfide form $\mathrm{MeS}_{2}$, oxysulfide form $\mathrm{MeS}_{x} \mathrm{O}_{y}$, which could be related to $\mathrm{Me}^{5+}$ form, and $\mathrm{Me}^{6+}$ form, which is typically associated with the metal oxide form. Unexpectedly, the spectrum of PAF-20-NiWS after the reaction in the presence of sulfur contains one additional phase that has signals with lower values of binding energy than those corresponding to the $\mathrm{WS}_{2}$ phase (Figure 5b). Assuming that the root of the above-mentioned phenomenon is in the sulfur deficiency in the new phase, we called it $\mathrm{WS}_{\mathrm{x}}$. The weight ratios of the resulting phases are listed in Table 2.

Figure 5a,b show the difference in the XPS spectra between the catalysts with the sulfidizing agent and without it. As it is clearly seen, in the case of experiments without sulfur, the predominant form is $\mathrm{Me}^{6+}$, whereas the addition of the sulfur leads to the other distribution of metal forms.

Table 2. X-ray photoelectron spectroscopy (XPS) data for the W4f and Mo3d levels.

\begin{tabular}{|c|c|c|c|c|c|}
\hline Catalyst & $\bar{L}, \mathrm{~nm}$ & $\operatorname{MeS}_{x}$ & $\mathrm{MeS}_{2}$ & $\operatorname{MeS}_{x} \mathrm{O}_{y}$ & $\mathrm{Me}^{6+}$ \\
\hline \multirow{3}{*}{ PAF-20-NiWS } & Binding eneroy $(\mathrm{eV})$ & \multirow{3}{*}{ - } & $32.64 \mathrm{f}_{7 / 2}$ & $33.54 f_{7 / 2}$ & $36.04 f_{7 / 2}$ \\
\hline & Binding energy $(\mathrm{ev})$ & & $34.24 \mathrm{f}_{5 / 2}$ & $35.34 f_{5 / 2}$ & $38.14 f_{5 / 2}$ \\
\hline & Weight fraction (\%) & & $3 \%$ & $3.5 \%$ & $93.5 \%$ \\
\hline \multirow{3}{*}{ PAF-20-NiWS + S } & \multirow{2}{*}{ Binding energy $(\mathrm{eV})$} & $30.74 f_{7 / 2}$ & $32.54 f_{7 / 2}$ & $33.54 f_{7 / 2}$ & $35.94 f_{7 / 2}$ \\
\hline & & $32.64 \mathrm{f}_{5 / 2}$ & $34.44 \mathrm{f}_{5 / 2}$ & $35.54 \mathrm{f}_{5 / 2}$ & $37.84 \mathrm{f}_{5 / 2}$ \\
\hline & Weight fraction (\%) & $12.7 \%$ & $40.3 \%$ & $7.0 \%$ & $40.0 \%$ \\
\hline \multirow{3}{*}{ PAF-20-NiMoS + S } & \multirow{2}{*}{ Binding energy $(\mathrm{eV})$} & \multirow{3}{*}{ - } & $229.04 f_{7 / 2}$ & $230.04 \mathrm{f}_{7 / 2}$ & $232.84 f_{7 / 2}$ \\
\hline & & & $232.14 \mathrm{f}_{5 / 2}$ & $233.64 \mathrm{f}_{5 / 2}$ & $235.74 f_{5 / 2}$ \\
\hline & Weight fraction (\%) & & $55 \%$ & $26.2 \%$ & $18.8 \%$ \\
\hline
\end{tabular}




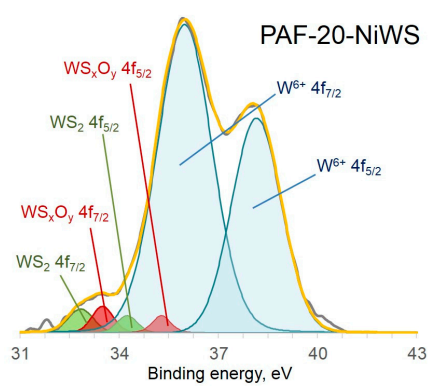

(a)

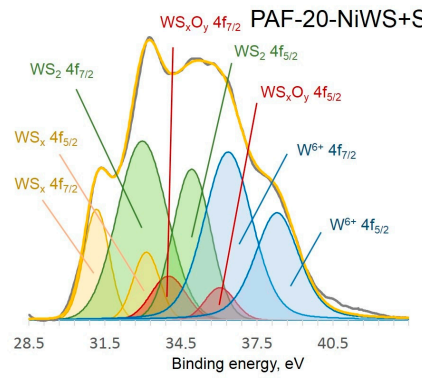

(b)

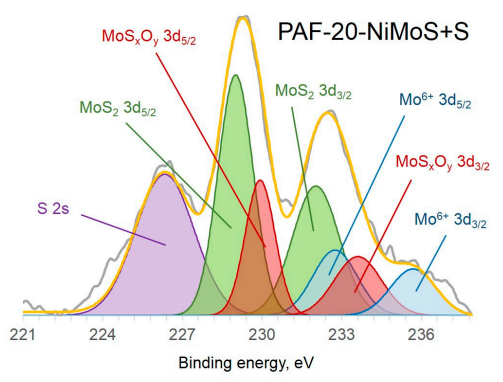

(c)

Figure 5. Deconvolution of W4f and Mo3d levels for catalysts after reaction: (a) PAF-20-NiWS; (b) PAF-20-NiWS, reaction in the presence of sulfur; and (c) PAF-20-NiMoS, reaction in the presence of sulfur.

Figure 5c presents the typical XPS spectra of molybdenum sulfide catalyst. According to the spectrum and Table 2, the share of $\mathrm{MoS}_{2}$ is $55 \%$, while the remaining $45 \%$ is in the $\operatorname{MoS}_{x} \mathrm{O}_{y}(26 \%)$ and $\mathrm{Mo}^{6+}(19 \%)$ forms. However, after experiments with the same reaction conditions, in the case of the NiWS catalyst, the share of the $\mathrm{Me}^{6+}$ form is $40 \%$ (Figure $5 \mathrm{~b}$ ), which is twice more than that for the NiMoS catalyst. This could be related to more facile transition from oxidized form to other forms for molybdenum in comparison with tungsten.

Figure 6 shows the representative 13C cross polarization/magic angle spinning nuclear magnetic resonance (CP/MAS NMR) spectra of PAF-NiMeS $(\mathrm{Me}=\mathrm{W}, \mathrm{Mo})$ before and after the reaction. The signals in the area of 110-150 ppm and near $66 \mathrm{ppm}$ correspond to the carbon atoms of the support, and signals at 14.2, 20.3, 24, and $57.4 \mathrm{ppm}$ are characteristic for $\mathrm{NBu}_{4}$ cations from the precursor salt. The spectrum of the catalyst after the reaction contains only characteristic PAF signals, indicating the full decomposition of tetraalkylammonium salt and the stability of the support during the reaction.
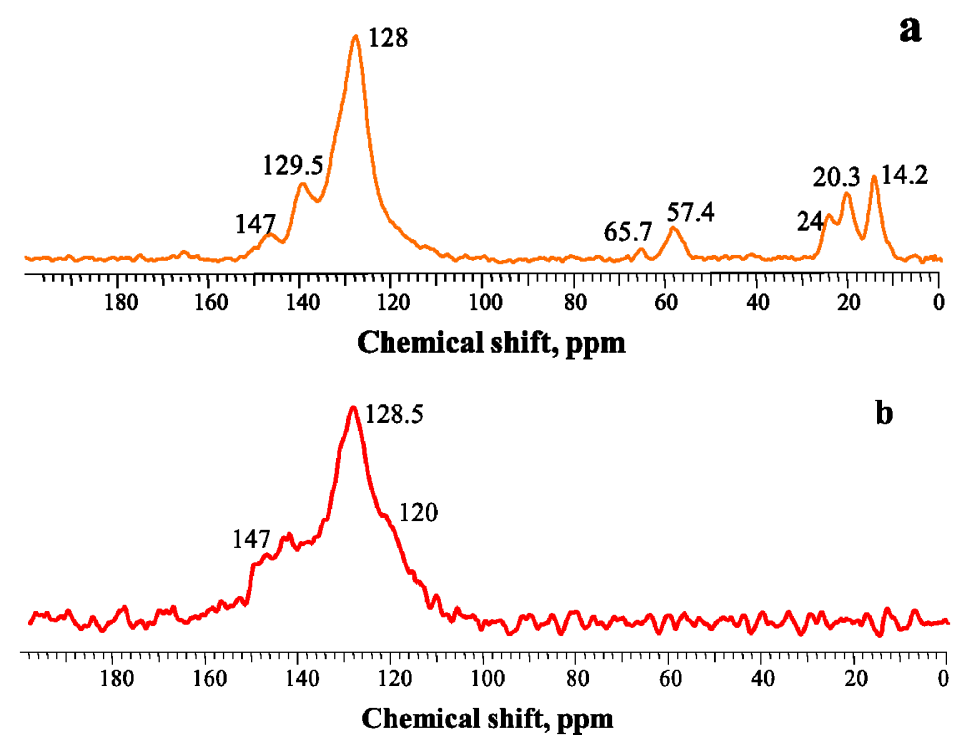

Figure 6. 13C CP/MAS NMR spectra of PAF-30-NiWS catalyst before (a) and after (b) the reaction.

\subsection{Catalytic Experiments}

The catalytic activity of synthesized materials was tested in the hydrogenation-hydrocracking reactions of naphthalene. The thermal stability of porous aromatic frameworks was discussed in recent works $[19,20]$; it was shown that in an air atmosphere PAFs start to decompose above 
$400{ }^{\circ} \mathrm{C}$, and, therefore, we assume the absence of full decomposition of catalyst supports under the experimental conditions. The PAFs themselves were found to have very low hydroprocessing activity, e.g., naphthalene conversion never exceeded $1 \%$. The activities of all catalysts were, nevertheless, corrected for the contribution of the support.

According to data in the literature, naphthalene conversion over sulfided catalysts takes place through a complex reaction scheme (Figure 7) [26]. The first step in the reaction mechanism includes hydrogenation of one benzene ring in naphthalene with tetralin formation. Further transformation of tetralin yields numerous products of aromatic ring hydrogenation, naphthenic ring isomerization, naphthenic ring opening, and cracking reactions.

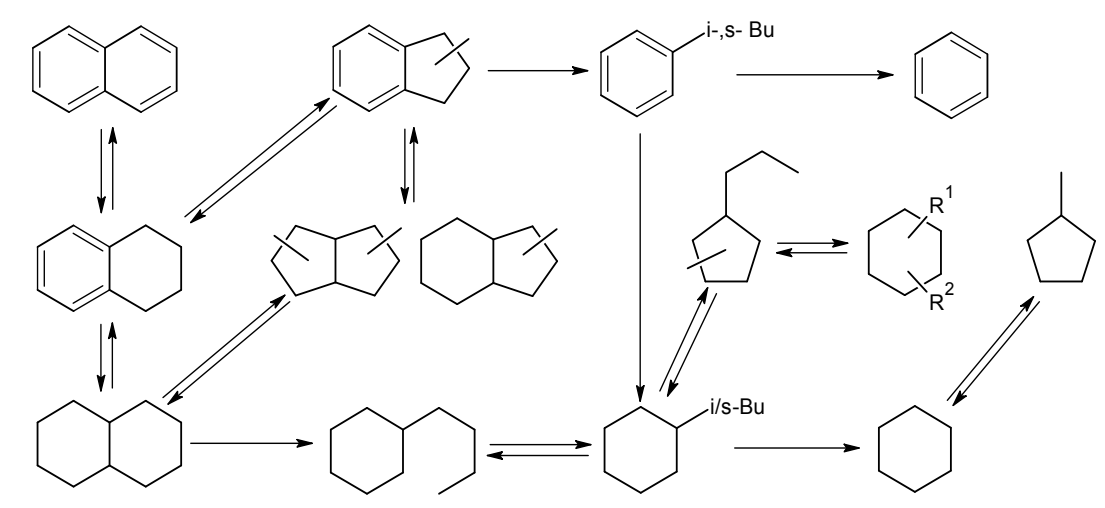

Figure 7. The proposed monomolecular reaction mechanism of naphthalene hydrogenationhydrocracking reactions.

The results of naphthalene hydrotreatment over PAF-NiMeS catalysts are summarized in Figure 8. In most cases, the predominant products are tetralin and decalines, instead of ring-opening and cracking derivatives through deep hydrogenation. The lack of other processes could be explained by the absence of acidity of the support: it is well-known that ring-opening and isomerization reactions occur through the protonated state of substrate, and cracking reactions are more likely for the products of ring-opening reactions than for bicyclic compounds, such as tetralin and indanes [26]. These results are in accordance with the results previously reported by Liu Chenguang et al. [27] for NiMoS nanoparticles on activated carbon and by Ferraz et. al. for NiMoS catalysts supported by alumina [4]. Furthermore, modifying benzene rings with even low-acidic OH-groups increases the yields of cracking products [23].

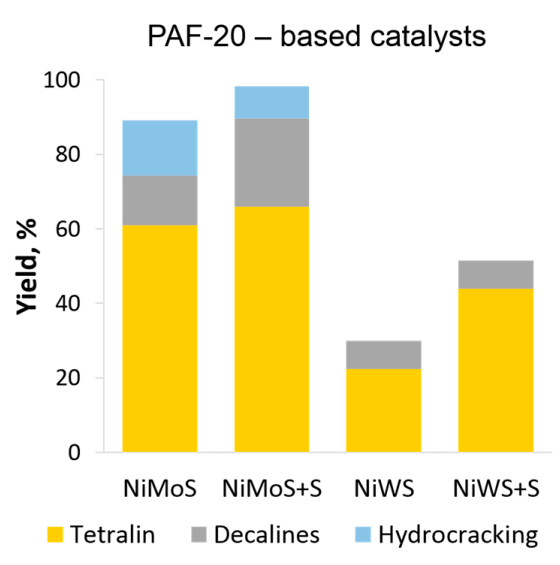

(a)

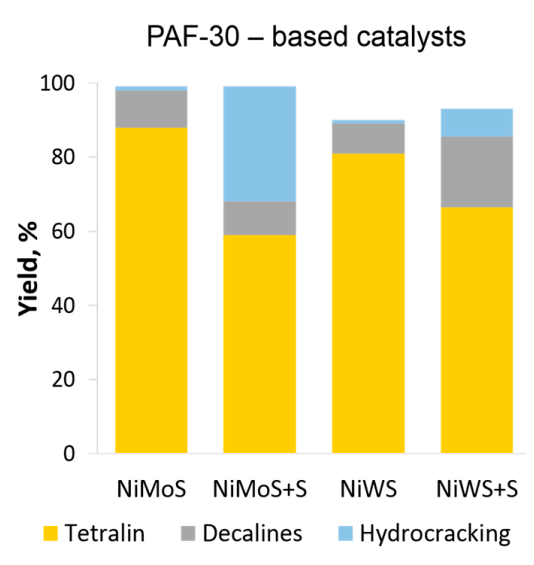

(b)

Figure 8. Product yield distribution for all catalytic systems: (a) PAF-20-based catalysts; and (b) PAF-30-based catalysts. 
It should be mentioned that, in all cases, biphenyl was revealed in the reaction products. The latter seems to be the product of partial framework destruction during the NiMeS-particles formation processes. To confirm this assumption the "blank" experiment without naphthalene was performed. Indeed, in this case the main reaction product was biphenyl. However, its quantity was as low as $1 \%-3 \%$ from all reaction products in experiments with naphthalene.

Additionally, as was expected, the addition of sulfur to the reaction media enhances the activity of the catalysts. We attribute it to the higher content of the catalytically-active sulfide phase in the case of experiments with the sulfur additive.

Figure 9 presents the dependence of the naphthalene conversion on the reaction time in the presence of PAF-30-NiMoS. The initial reaction rate is lower than that after three hours after the beginning of reaction. Probably, such an induction period of reaction related to the processes of active phase formation.

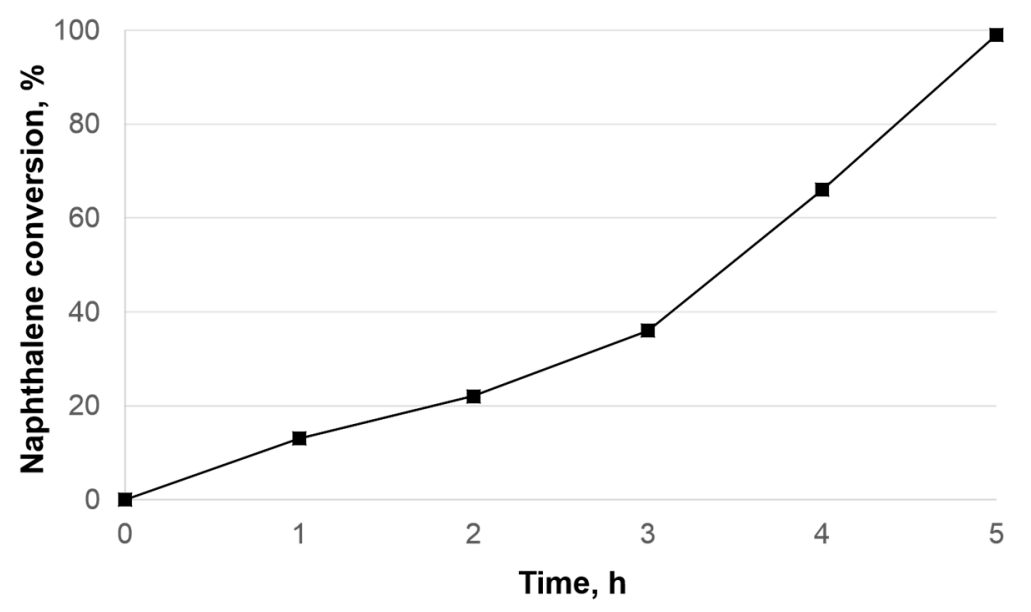

Figure 9. Kinetic curve of naphthalene hydrogenation in presence of PAF-30-NiMoS catalyst. Reaction conditions: $380{ }^{\circ} \mathrm{C}, 5 \mathrm{MPa} \mathrm{H}_{2}$, catalyst (50 mg), naphthalene $(80 \mathrm{mg})$, benzene $(2 \mathrm{~mL})$.

\section{Experimental}

\subsection{Materials}

All starting materials were purchased from commercial suppliers and used as received: benzene1,4-diboronic acid ( $\geq 95.0 \%$, Sigma-Aldrich, Schnelldorf, Germany); 4,4'-Biphenyldiboronic acid (95.0\%, ABCR Chemie Rus, Moscow, Russia); trityl chloride (purum, $\geq 97.0 \%$, Sigma-Aldrich); aniline (ReagentPlus ${ }^{\circledR}, 99 \%$, Sigma-Aldrich); hypophosphorous acid solution (50 wt. \% in water, Sigma-Aldrich); bromine (reagent grade, Sigma-Aldrich); palladium (II) acetate (reagent grade, 98\% Sigma-Aldrich); triphenylphosphine (ReagentPlus ${ }^{\circledR}, 99 \%$, Sigma-Aldrich); sodium tungstate dihydrate (high purity grade, Reakhim, Moscow, Russia); sodium molybdate dihydrate (high purity grade, Reakhim); nickel(II) chloride hexahydrate (puriss. p.a., $\geq 98 \%$, Sigma-Aldrich); ammonium hydroxide solution (analytical grade, Reakhim); tetrabutylammonium bromide (ReagentPlus grade, $\geq 99 \%$, Sigma-Aldrich); naphthalene (97\%, Sigma-Aldrich). Sigma-Aldrich reagents were from Schnelldorf, Bavaria, Germany; Reakhim reagents were from Staraya Kupavna, Moscow region, Russia. The purification of solvents was performed according to standard methods. Porous aromatic frameworks were synthesized as described earlier [28].

\subsection{Synthesis of Precursors}

The ammonium thiomolybdate and thiotungstate were prepared as described elsewhere [29]. The tetraalkylammonium nickel tetrathyometallate salts were obtained by the previously described method [23]. 
The immobilization of thiosalts on the porous aromatic frameworks was performed by impregnation of PAFs with $\left[(\mathrm{n}-\mathrm{Bu})_{4} \mathrm{~N}_{2}\left[\mathrm{Ni}\left(\mathrm{MeS}_{4}\right)_{2}\right](\mathrm{Me}=\mathrm{W}, \mathrm{Mo})\right.$ with a minimum amount of THF and further drying using the rotary evaporator. Molybdenum or tungsten content in the final product was $15 \%$ of the mass. The typical procedure includes the dissolving of $1.35 \mathrm{~g}$ of $\left[(\mathrm{n}-\mathrm{Bu})_{4} \mathrm{~N}\right]_{2}\left[\mathrm{Ni}\left(\mathrm{WS}_{4}\right)_{2}\right]$ in $100 \mathrm{~mL}$ of THF and further addition to the solution of $1.5 \mathrm{~g}$ of PAF. The suspension is stirred for $12 \mathrm{~h}$ and dried under reduced pressure using a rotary evaporator.

The final precursors were labeled as PAF-20-NiWS, PAF-20-NiMoS, PAF-30-NiWS, and PAF-30-NiMoS, where PAF-20 and PAF-30 are names of the frameworks and NiWS and NiMoS are abbreviations of corresponding tetraalkylammonium salt.

\subsection{Characterization of Catalysts}

The textural properties of the prepared in situ catalysts were determined from high-resolution transmission electron microscopy microphotographs, obtained on a JEOL JEM-2100 microscope (JEOL Ltd., Akishima, Tokyo, Japan), equipped with an energy dispersive JED-2300 spectrometer (JEOL Ltd., Akishima, Tokyo, Japan), with a $0.14 \mathrm{~nm}$ lattice fringe resolution, an accelerating voltage of $200 \mathrm{kV}$ and camera length of $400 \mathrm{~mm}$ ( $\mathrm{L} \lambda$ close to 10). The samples were prepared on a perforated carbon film mounted on a copper grid, and 10-15 representative micrographs were obtained for each catalyst in high-resolution mode. Typically, the lengths of at least 400 slabs were measured for each catalyst.

The XPS analysis of the catalysts was performed on a Kratos Axis Ultra DLD instrument (Kratos, Manchester, UK) using excitation with $\mathrm{Al} \mathrm{K \alpha}$ X-ray radiation at $1486.6 \mathrm{eV}$. The calibration of photoelectron peaks was based on the $\mathrm{C} 1 \mathrm{~s}$ line with a binding energy of $284.7 \mathrm{eV}$. The transmission energy of the energy analyzer was $160 \mathrm{eV}$ (a survey spectrum) or $40 \mathrm{eV}$ (individual lines).

The solid-state NMR spectra were measured on a Varian NMR Systems instrument with a resonance frequency of $125 \mathrm{MHz}$ for 13C cross polarization (CP) MAS NMR spectra in impulse mode at a spinning speed of $10 \mathrm{kHz}$.

\subsection{Catalytic Testing Procedure}

The reaction was carried out in a steel reactor equipped with stir bar at $380{ }^{\circ} \mathrm{C}$ under a $5 \mathrm{MPa} \mathrm{H}_{2}$ pressure. The stir bar, the catalyst $(50 \mathrm{mg})$, naphthalene $(80 \mathrm{mg})$, sulfur $(50 \mathrm{mg}$, in several experiments), and benzene $(2 \mathrm{~mL})$ were introduced into the reactor. Then, the reactor was sealed and pressurized with $\mathrm{H}_{2}$ at the demanded pressure. The mixture was stirred at $380{ }^{\circ} \mathrm{C}$ and after the reaction was finished, the reactor was cooled down and depressurized. All of the reaction products were analyzed by GC analysis at a ChromPack CP9001 apparatus (Chrompack (acquired by Agilent Technologies), Middelburg, The Netherlands) equipped with FID and a semi-polar SE-30 100\% dimethylpolysiloxane column (30 m $\times 0.2 \mathrm{~mm}$ ID, $0.2 \mu \mathrm{m}$ film); nitrogen was used as a carrier gas, with a flow rate of $30 \mathrm{~mL} / \mathrm{min}$. Chromatograms were calculated using the program Maestro 1.4 (Version 1.4, Chrompack, Middelburg, The Netherlands, 1994).

\section{Conclusions}

The first example of using of porous aromatic frameworks as support for sulfide catalysts by in situ decomposition of $\left[(\mathrm{n}-\mathrm{Bu})_{4} \mathrm{~N}_{2}\left[\mathrm{Ni}\left(\mathrm{MeS}_{4}\right)_{2}\right](\mathrm{Me}=\mathrm{W}, \mathrm{Mo})\right.$ was considered. The properties of catalysts in hydrogenation of naphthalene were studied. The following principles were found:

1) The porous properties of the support have an impact on the performance of the catalysts. It was revealed that catalysts supported by the framework with a larger pore size were more active than those based on the framework with a smaller pore size;

2) Typically, in the reactions without the additive of sulfur, the predominant phases on a particle surface are $\mathrm{Mo}^{6+}$ and $\mathrm{W}^{6+}$; the addition of sulfur enhanced the catalytic performance of the systems by conversion of the $\mathrm{Me}^{6+}$ form to the more active $\mathrm{MeS}_{2}$ form; 
3) Molybdenum catalysts were found to be more active in the presence of sulfur than tungsten catalysts due to the more facile transition from the oxide form to the sulfide form.

Acknowledgments: The study was supported by the Russian Science Foundation within a framework of Project N 15-19-00099.

Author Contributions: Eduard Karakhanov and Anton Maximov conceived and designed the experiments; Leonid Kulikov performed the experiments; Anna Zolotukhina analyzed the data; Yulia Kardasheva, Maria Vinnikova and Andrey Ivanov contributed reagents/materials/analysis tools; Leonid Kulikov wrote the paper.

Conflicts of Interest: The authors declare no conflict of interest.

\section{References}

1. Farag, H.; Whitehurst, D.D.; Mochida, I. Synthesis of active hydrodesulfurization carbon-supported Co-Mo catalysts. Relationships between preparation methods and activity/selectivity. Ind. Eng. Chem. Res. 1998, 37, 3533-3539. [CrossRef]

2. Álvarez, L.; Berhault, G.; Alonso-Nuñez, G. Unsupported NiMo sulfide catalysts obtained from nickel/ammonium and nickel/tetraalkylammonium thiomolybdates: Synthesis and application in the hydrodesulfurization of dibenzothiophene. Catal. Lett. 2008, 125, 35-45. [CrossRef]

3. Díaz de León, J.N.; Picquart, M.; Massin, L.; Vrinat, M.; de los Reyes, J.A. Hydrodesulfurization of sulfur refractory compounds: Effect of gallium as an additive in $\mathrm{NiWS} / \gamma-\mathrm{Al}_{2} \mathrm{O}_{3}$ catalysts. J. Mol. Catal. A Chem. 2012, 363-364, 311-321. [CrossRef]

4. Ferraz, S.G.A.; Zotin, F.M.Z.; Araujo, L.R.R.; Zotin, J.L. Influence of support acidity of NiMoS catalysts in the activity for hydrogenation and hydrocracking of tetralin. Appl. Catal. A Gen. 2010, 384, 51-57. [CrossRef]

5. Nikulshin, P.A.; Minaev, P.P.; Mozhaev, A.V.; Maslakov, K.I.; Kulikova, M.S.; Pimerzin, A.A. Investigation of co-effect of 12-tungstophosphoric heteropolyacid, nickel citrate and carbon-coated alumina in preparation of NiW catalysts for HDS, HYD and HDN reactions. Appl. Catal. B Environ. 2015, 176-177, 374-384. [CrossRef]

6. Sizova, I.A.; Serdyukov, S.I.; Maksimov, A.L. Nickel-tungsten sulfide aromatic hydrocarbon hydrogenation catalysts synthesized in situ in a hydrocarbon medium. Pet. Chem. 2015, 55, 470-480. [CrossRef]

7. Brito, J.N.L.; Severino, F.; Ninoska Delgado, N.; Laine, J. Hds activity of carbon-supported Ni-Mo catalysts derived from thiomolybdate complexes. Appl. Catal. A Gen. 1998, 173, 193-199. [CrossRef]

8. Wilkinson, K.; Merchán, M.D.; Vasudevan, P.T. Characterization of supported tungsten sulfide catalysts ex ammonium tetrathiotungstate. J. Catal. 1997, 171, 325-328. [CrossRef]

9. Dutta, R.P.; Schobert, H.H. Catalytic conversion of polycyclic aromatic hydrocarbons hydrogenation/ dehydrogenation of polycyclic aromatic hydrocarbons using ammonium tetrathiomolybdate as catalyst precursor. Catal. Today 1996, 31, 65-77. [CrossRef]

10. Brito, J.L.; Ilija, M.; Hernández, P. Thermal and reductive decomposition of ammonium thiomolybdates. Thermochim. Acta 1995, 256, 325-338. [CrossRef]

11. Poisot, M.; Bensch, W. Decomposition of tetraalkylammonium thiotungstates characterized by thermoanalysis, mass spectrometry, X-ray diffractometry and scanning electron microscopy. Thermochim. Acta 2007, 453, 42-51. [CrossRef]

12. Minaev, P.P.; Nikulshin, P.A.; Kulikova, M.S.; Pimerzin, A.A.; Kogan, V.M. NiWS $/ \mathrm{Al}_{2} \mathrm{O}_{3}$ hydrotreating catalysts prepared with 12-tungstophosphoric heteropolyacid and nickel citrate: Effect of Ni/W ratio. Appl. Catal. A Gen. 2015, 505, 456-466. [CrossRef]

13. Lauritsen, J.V.; Nyberg, M.; Nørskov, J.K.; Clausen, B.S.; Topsøe, H.; Lægsgaard, E.; Besenbacher, F. Hydrodesulfurization reaction pathways on $\mathrm{MoS}_{2}$ nanoclusters revealed by scanning tunneling microscopy. J. Catal. 2004, 224, 94-106. [CrossRef]

14. Vázquez, P.; Pizzio, L.; Blanco, M.; Cáceres, C.; Thomas, H.; Arriagada, R.; Bendezú, S.; Cid, R.; García, R. $\mathrm{NiMo}(\mathrm{W})$-based hydrotreatment catalysts supported on peach stones activated carbon. Appl. Catal. A Gen. 1999, 184, 303-313. [CrossRef]

15. Vissers, J.P.R.; Scheffer, B.; de Beer, V.H.J.; Moulijn, J.A.; Prins, R. Effect of the support on the structure of Mo-based hydrodesulfurization catalysts: Activated carbon versus alumina. J. Catal. 1987, 105, 277-284. [CrossRef] 
16. Topsøie, H.; Candia, R.; Topsøe, N.-Y.; Clausen, B.S.; Topsøe, H. On the state of the Co-Mo-S model. Bull. Soc. Chim. Belg. 1984, 93, 783-806. [CrossRef]

17. Hinnemann, B.; Nørskov, J.K.; Topsøe, H. A density functional study of the chemical differences between type I and type II MoS 2 -based structures in hydrotreating catalysts. J. Phys. Chem. B 2005, 109, 2245-2253. [CrossRef] [PubMed]

18. Topsøe, H.; Clausen, B.S.; Massoth, F.E. Hydrotreating catalysis. In Catalysis: Science and Technology; Anderson, J.R., Boudart, M., Eds.; Springer Berlin Heidelberg: Berlin/Heidelberg, Germany, 1996; pp. 1-269.

19. Ben, T.; Ren, H.; Ma, S.; Cao, D.; Lan, J.; Jing, X.; Wang, W.; Xu, J.; Deng, F.; Simmons, J.M.; et al. Targeted synthesis of a porous aromatic framework with high stability and exceptionally high surface area. Angew. Chem. Int. Ed. 2009, 48, 9457-9460. [CrossRef] [PubMed]

20. Yuan, Y.; Sun, F.; Ren, H.; Jing, X.; Wang, W.; Ma, H.; Zhao, H.; Zhu, G. Targeted synthesis of a porous aromatic framework with a high adsorption capacity for organic molecules. J. Mater. Chem. 2011, 21, 13498-13502. [CrossRef]

21. Maximov, A.; Zolotukhina, A.; Murzin, V.; Karakhanov, E.; Rosenberg, E. Ruthenium nanoparticles stabilized in cross-linked dendrimer matrices: Hydrogenation of phenols in aqueous media. ChemCatChem 2015, 7, 1197-1210. [CrossRef]

22. Berhault, G.; Cota Araiza, L.; Duarte Moller, A.; Mehta, A.; Chianelli, R.R. Modifications of unpromoted and cobalt-promoted $\mathrm{MoS}_{2}$ during thermal treatment by dimethylsulfide. Catal. Lett. 2002, 78, 81-90. [CrossRef]

23. Karakhanov, E.; Boronoev, M.; Ignatyeva, V.; Maximov, A.; Filippova, T.; Kardasheva, Y. Hydroprocessing of aromatics using sulfide catalysts supported on ordered mesoporous phenol-formaldehyde polymers. J. Inorg. Organomet. Polym. Mater. 2016, 1-6. [CrossRef]

24. Ishutenko, D.; Nikulshin, P.; Pimerzin, A. Relation between composition and morphology of K(Co)MoS active phase species and their performances in hydrotreating of model FCC gasoline. Catal. Today 2016, 271, 16-27. [CrossRef]

25. Li, M.; Li, H.; Jiang, F.; Chu, Y.; Nie, H. The relation between morphology of (Co) $\mathrm{MoS}_{2}$ phases and selective hydrodesulfurization for CoMo catalysts. Catal. Today 2010, 149, 35-39. [CrossRef]

26. Lemberton, J.L.; Baudon, A.; Guisnet, M.; Marchal, N.; Mignard, S. Hydrocracking of $C_{10}$ hydrocarbons over a sulfided NiMo/Y zeolite catalyst. In Studies in Surface Science and Catalysis; Froment, G.F., Delmon, B., Grange, P., Eds.; Elsevier: Amsterdam, The Netherlands, 1997; Volume 106, pp. 129-136.

27. Liu, C.; Wang, S.; An, C.; Yan, F.; He, J.; Wang, Z.; Zhang, Q.-H. Oil-soluble Ni-Mo sulfide nanoparticles and their hydrogenation catalytic properties. Pet. Sci. 2013, 10, 571-576. [CrossRef]

28. Maximov, A.; Zolotukhina, A.; Kulikov, L.; Kardasheva, Y.; Karakhanov, E. Ruthenium catalysts based on mesoporous aromatic frameworks for the hydrogenation of arenes. React. Kinet. Mech. Catal. 2016, 117, 729-743. [CrossRef]

29. McDonald, J.W.; Friesen, G.D.; Rosenhein, L.D.; Newton, W.E. Syntheses and characterization of ammonium and tetraalkylammonium thiomolybdates and thiotungstates. Inorg. Chim. Acta 1983, 72, 205-210. [CrossRef]

(C) 2016 by the authors; licensee MDPI, Basel, Switzerland. This article is an open access article distributed under the terms and conditions of the Creative Commons Attribution (CC-BY) license (http://creativecommons.org/licenses/by/4.0/). 